\title{
An Automatic Left Ventricle Segmentation on Echocardiogram Exams via Morphological Geodesic Active Contour with Adaptive External Energy
}

\author{
Aldísio G. Medeiros ${ }^{1}$, Francisco H. S. Silva ${ }^{1}$, Elene F. Ohata ${ }^{1}$, \\ Solon A. Peixoto ${ }^{1}$, Pedro P. Rebouças Filho ${ }^{1}$ \\ ${ }^{1}$ Laboratório de Processamento de Imagens, Sinais e Computação Aplicada, Instituto Federal do Ceará, Fortaleza, CE, Brasil \\ Email: aldisio.medeiros@lapisco.ifce.edu.br;herculessilva@lapisco.ifce.edu.br;pedrosarf@ifce.edu.br \\ *Corresponding Author: Pedro P. Rebouças Filho, Email: pedrosarf@ifce.edu.br
}

How to cite this paper: Medeiros, Aldísio G.; Silva, Francisco H. S.; Ohata, Elene F. Peixoto, Solon A. and Rebouças Filho, Pedro P. (2019) An Automatic Left Ventricle Segmentation on Echocardiogram Exams via Morphological Geodesic Active Contour with Adaptive External Energy. Journal of Artificial Intelligence and Systems, 1, 77-95.

https://doi.org/10.33969/AIS.2019.11005

Received: September 3, 2019

Accepted: September 19, 2019

Published: October 9, 2019

Copyright (C) 2019 by author(s) and Institute of Electronics and Computer. This work is licensed under the Creative Commons Attribution International License (CC BY 4.0).

http://creativecommons.org/licenses/by/4.0/

(c) (7) Open Access

\begin{abstract}
This work proposes a new adaptive approach to left ventricle segmentation based on a non-parametric adaptive active contour method called Fast Morphological Geodesic Active Contour (FGAC) combined with adaptive external energy via deep learning model. The evaluation methodology considered echocardiogram exams obtained from volunteers. Beyond the manual segmentations made by two specialists medical as ground truth. The new approach is compared with three other segmentation methods, also based on the active contour method: pSnakes, radial snakes with derivative (RSD), and radial snakes with Hilbert energy (RSH). The FGAC combined with adaptive external energy showed better Precision $(99.53 \%, 99.72 \%)$ against RSD $(99.46 \%, 99.68 \%)$, RSH $(99.51 \%, 99.71 \%)$ and pSnakes $(99.52 \%, 99.72 \%)$. Besides, it achieved a relevant Jaccard similarity index $(67.40 \%, 62.02 \%)$, and promising accuracy $(98.64 \%, 98.46 \%)$. Even though the metrics differences are low, the proposed approach is fully automatic. Therefore, these results suggest the potential of the proposed approach to aid medical diagnosis systems in echocardiology.
\end{abstract}

\section{Keywords}

Active contours, Image Segmentation, deep learning, FGAC, Echocardiogram, Artificial Intelligence

\section{Introduction}

The cardiac pathologies have occupied worrying positions among the diseases that most cause deaths worldwide. In 2017, were registered 253 deaths per 100000 people in United States [14], 67 in Germany, reaching most rates in Ukraine with 356 cases [32]. In Brazil, mortality rate by cardiovascular disease reached 318 cases in same period, surpassing deaths by cancer and respiratory diseases [10].

Important information to medical diagnosis of cardiovascular disease (CVD) can be obtained from left-ventricle (LV). Attributes as ventricle area and volume are important to diagnostic of anomalies such as cardiac ischemia or ventricle dysfunction, beyond to guide the specialist in planning and execution of surgical procedure [39]. 
In this sense, image processing techniques applied in diagnosis systems of echocardiogram exams has been explored [4]. Among the applied techniques for medical image segmentation can be mentioned mathematics morphology [30], region growing with local thresholds [8], beyond watershed transform [3]. However, echocardiogram images have a low contrast and the image objects boundaries do not contribute to appropriate segmentation. According [37], traditional techniques do not obtain consistent segmentations to regions with low contrast parenchyma leading to inappropriate results.

Thus, approaches inspired in deformable models has been highlighted makes the segmentation more tolerant of noises such as methods based on the active contour methods (ACM), also known as snakes. For instance, traditional snakes [24], Radial snakes with derivative (RSD), Radial snakes with Hilbert energy (RSH) [1], and pSnakes based on polar coordinates [9].

The segmentation inspired in ACM is formed by segments of lines that evolve, forming a curve in the image domain. The curve is adapted according to the shape of the region near the starting point, evolving to the edges of the object of interest. The adaptation process aims to minimize a total energy formed by energies that depend on the active contour geometry, the internal energy, and image characteristics that form the external energy [24].

Traditionally, ACMs need a definition of starting contour, and segmentation result can change according to the place where it was defined. When the starting contour is applied in regions with concavity, protrusions or bifurcations, it can lead to unexpected results [37]. In this case, a possible solution is to consider the density of the internal structures of the cardiac muscle tissue, guiding the segmentation method in order to reduce the influence of the noise.

The main noise present in the echocardiogram exams, known as speckle noise, is a multiplicative noise and it is proportional to the intensity of the signal received by the probe [4]. This noise produces a visual effect with a grainy texture, and it can difficult the interpretation of the region on the image, reducing the definition of the edges that delimit cardiac muscle. Thus, this work aims to develop a new adaptive approach, with automatic initialization using the recent deep learning method called Mask R-CNN, proposed by He in [19]. The advantage of this model over others is the ease in which it can adapt to different tissues of cardiac parenchyma. Furthermore, this work provides a new approach to segmentation of left-ventricle based on recent method FGAC proposed by Medeiros et al. [29] adding a new adaptive external energy, in addition to comparing it with other ventricle segmentation methods.

This paper is organized as follows: Section 2 presents a literature review of the main topics related to this work, in Section 3, we present the algorithms and classification models which give basis for the proposed methodology. In Section 4, all the details concerning the methodology used here are discussed. The results and discussions are presented in Section 5, which leads to the conclusions in Section 6.

\section{Left-Ventricle Segmentation Overview}

In the past several decades, many segmentation methods have been proposed to address LV segmentation. A comprehensive review can be found in [35]. These approaches may be classified into three groups: model based approaches [31], image feature based methods [7] and deformable models $[1,9,4]$

Some approaches are based in statistic model, for instance, K-Means clustering algorithm is combined with mean filtering in order to identify starting point of active contour [31]. This method was combined with traditional active contour [24] for detecting ventricle boundaries. However, the model presented limitation for regions with cavities leading results with unwanted regions.

Other approaches are based in extracted features of image, such as shape and textures. Active Appearance Models, developed by Cootes et al. [7], combine a model of shape 
variation with a model of texture variation defining a set of dots that can represent the main features of interest objects.

Active contours models [24], also known as snakes, have often been used for the processing of medical images. A review of these approaches and their medical application is given in [4]. The main recent researches aim ventricle segmentation using deformable models with the new design of the external energy [37, 1].

The recent approaches using polar coordinates for ventricle segmentation [1], designed a new external energy, this method is called pSnake. This technique is based on a nodes pair called control nodes for detection edges of interest region. However, this method may not have a behavior adequate when the desired object has a smooth edge, making it difficult to detect peaks and consequently to define the boundary. Best results were for circular and continuous forms.

Other approach, proposed by Guo et al. [16], presents an automatic myocardial segmentation method based on active contours and measures of neutrosophic similarity, a neutrophysiologic active contour model (NACM). The method uses a tissue clustering approach to detect the left ventricle region. Performance evaluation is compared to manual segmentation by an experienced radiologist. The evaluation metrics used were Hausdorff Distance and Mean Distance.

More recent, Medeiros et al. [29] proposed a segmentation method called Fast Morphological Geodesic Active Contour (FGAC), this approach is based on geometric active contour. Inspired on equivalencies originally proposed by Neila et al. [27], this method is built over mathematical morphology operators. The FGAC method is differentiated by its potential to find an initial Level Set more near to desired structure edges, this feature improves the convergence time, accelerating the segmentation task.

\section{Materials}

This section describes the main literature methods that support the left ventricular segmentation methodology proposed in this paper.

\subsection{Feature Extraction}

The classification stage is based on the statistical and spatial distribution of the samples. In this respect, the representative characteristics of each class need to be extracted. This section presents three features extractors already consolidated in the literature.

Haralick et al. [17] proposed a statistical analysis according to co-ocorrency on gray levels in the image. This method is called Gray-Level Co-Occurrence Matrix (GLCM) and identifies the spacial influences of pixels concerning their grayscale. This method shows that the use of statistical methods provides a dependent association between pixels. From this co-occurrence matrix, it is possible to extract fourteen original descriptors. This descriptors ability to represent, among the features, the angular second moment and entropy are presented on Equation 1 and 2, respectively,

$$
\begin{gathered}
\sum_{i} \sum_{j} p(i, j)^{2} \\
-\sum_{i} \sum_{j} p(i, j) \log (p(i, j))
\end{gathered}
$$

where $p$ is central pixel, $i$ and $j$ are indexes according to image height and width. 
HU et al. [21] developed a model that uses central moments to become the method invariant to scale and rotation changes. This method, known as HU moments, describes a feature extraction family composed of seven moments; each one is invariable to size, rotation, and translation operations. Equation 3 shows the relation between central moment and normalized moment. This normalized moment can be obtained by the central moment, $\mu_{p q}$, divides by an exponential of the area, $\mu_{00}$, to obtain the normalized central moment, $\eta_{p q}$

$$
\eta_{p q}=\frac{\mu_{p q}}{\mu_{00}^{\alpha}}
$$

where

$$
\alpha=\frac{p+q}{2} ; \forall p+q \geq 2
$$

Then, the seven moments are calculated by using their result as the image attributes. These seven moments are described as

- $I_{1}=\eta_{20}+\eta_{02}$

- $I_{2}=\left(\eta_{20}-\eta_{02}\right)^{2}+4 \eta_{11}^{2}$

- $I_{3}=\left(\eta_{30}-3 \eta_{12}\right)^{2}+\left(3 \eta_{21}-\eta_{03}\right)^{2}$

- $I_{4}=\left(\eta_{30}+\eta_{12}\right)^{2}+\left(\eta_{21}+\eta_{03}\right)^{2}$

- $I_{5}=\left(\eta_{30}-3 \eta_{12}\right)\left(\eta_{30}+\eta_{12}\right)\left[\left(\eta_{30}+\eta_{12}\right)^{2}-3\left(\eta_{21}+\eta_{03}\right)^{2}\right]+\left(3 \eta_{21}-\eta_{03}\right)\left(\eta_{21}+\right.$ $\left.\eta_{03}\right)\left[3\left(\eta_{30}+\eta_{12}\right)^{2}-\left(\eta_{21}+\eta_{03}\right)^{2}\right]$

- $I_{6}=\left(\eta_{20}-\eta_{02}\right)\left[\left(\eta_{30}+\eta_{12}\right)^{2}-\left(\eta_{21}+\eta_{03}\right)^{2}\right]+4 \eta_{11}\left(\eta_{30}+\eta_{12}\right)\left(\eta_{21}+\eta_{03}\right)$

- $I_{7}=\left(3 \eta_{21}-\eta_{03}\right)\left(\eta_{30}+\eta_{12}\right)\left[\left(\eta_{30}+\eta_{12}\right)^{2}-3\left(\eta_{21}+\eta_{03}\right)^{2}\right]-\left(\eta_{30}-3 \eta_{12}\right)\left(\eta_{21}+\right.$ $\left.\eta_{03}\right)\left[3\left(\eta_{30}+\eta_{12}\right)^{2}-\left(\eta_{21}+\eta_{03}\right)^{2}\right]$

The SCM feature extractor proposed by Ramalho et al. [36] focused on identifying object structures by applying a highlight function $K$ to it. The $K$ function must be set according to the characteristics of images that belong to the problem. The current $K$ highlight as a filter where the most relevant features of the image are highlighted. SCM performs pixel-by-pixel analysis by combining of a pixel in the original image concerning its neighboring pixels in the $K$-filtered filtered image, using some neighborhood criteria. Finally, this matrix is combined with its transposed matrix. The resulting symmetric matrix is the matrix from which SCM attributes can be extracted.

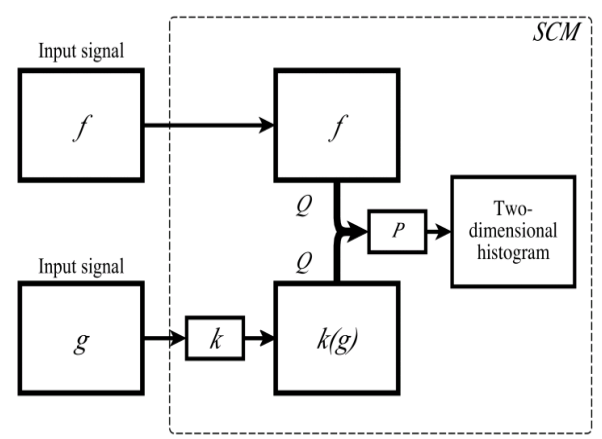

Figure 1. Generic SCM model for two input signals. The input signal is filtered by applying a $K$ function. Next, the two-dimensional SCM histogram is created from the original signal combined with the modified signal $k(g)$ [36].

\subsection{Classifiers}

In this section is described the machine learning techniques used to classify the features extracted by the traditional methods. 


\subsubsection{Naive Bayes}

Bayesian Classifier is based on input data statistical analysis. The classifications are based on the probability distribution of each sample to a specific class, considering that this class has the most probability of be associated with the sample [43]. Bayes Theory inspires this model, and it supposes that no exist dependencies among the features, according to the value of posterior probability and conditional probability.

\subsubsection{K-Nearest Neighbor}

K-Nearest Neighbor (KNN) is a machine learning method proposed by [13], that fall in the supervised category. It works by determining the class to which a sample belongs by comparing the features of the $\mathrm{k}$ nearest neighbors that were acquired in a previous training step. The variable $k$ represents the number of samples of the training set that possess the closest features to the sample being classified. Still regarding the variable $k$, there is not a standard value for it, but, in general, even numbers are avoided to prevent a drawn situation in which the sample could be classified in two classes at the same time.

\subsubsection{Multilayer Perceptron - MLP}

Multilayer Perceptron (MLP) is a neural network architecture formed by multiple layers with perceptron neurons. Input data vector is introduced to first layer where each feature is computed and each neuron contributes for transformation of input space to a linearly separable space and thus to classify the object to its specific class [18]. Learning technique is supervisioned through backpropagation algorithm where the errors calculated at last layer are retro propagated to adjust the hidden layers [18]. Therefore, through this procedure, a solution to samples in the input vector is presented on the output layer.

\subsection{4. $\mathrm{RBF}$}

A Radial Basis Function (RBF) network is a particular type of neural network that provides promising results in its use as a nonlinear classifier. Unlike MLP networks, the approach of RBF networks is more intuitive; the network ranks by measuring the similarity of the input data. The hidden layer neuron has a centroid, when new data is presented, each neuron of the intermediate layer calculates the Euclidean distance between the data and its centroid. A typical architecture of an RBF network, consisting of an input vector, a hidden layer with radial base function-activated neurons (Gaussian function), and an output layer that uses a linear activation function [34].

\subsubsection{ELM}

The Extreme Learning Machine (ELM) has the structure of an artificial neural network with only a single hidden layer. Described initially by Huang et al. [22], the ELM has a similar structure to the other Artificial Neural Networks, but the training principle is not based on the descending gradient. Besides, the method does not use the backpropagation algorithm, which makes the classifier robust to slow convergence and convergence to local minimal. ELM training assigns random values to the weight matrix of the hidden layer neurons. The matrix of the output layer is defined from the labels and by the resolution of a linear system. The output hidden layer is directed to the output layer producing a linear system where the solution can be obtained through the generalized inverse Moore-Penrose matrix [41]. This latter matrix is known as the features matrix. 


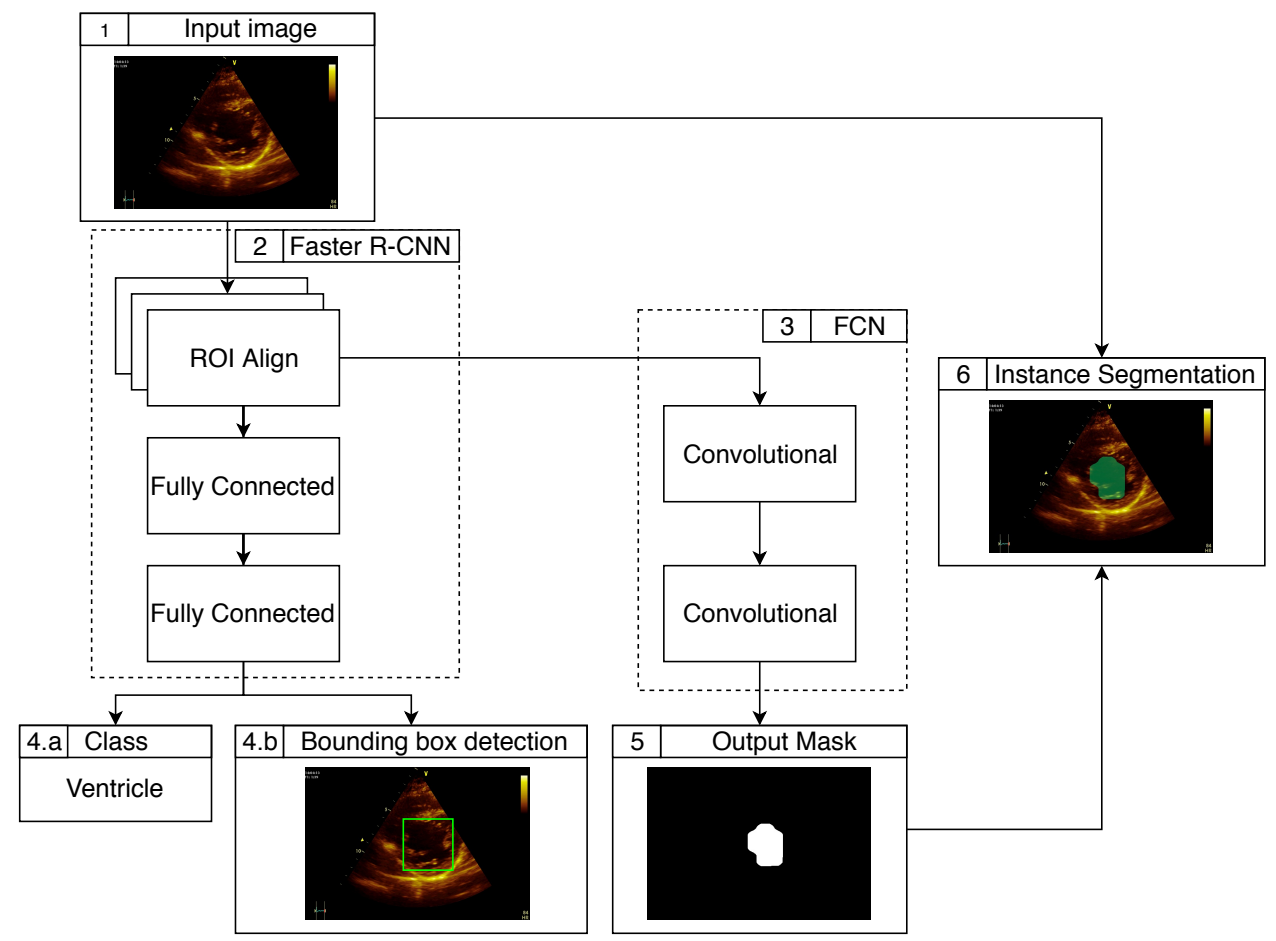

Figure 2. Diagram with Mask R-CNN architecture adapted for classification of left ventricle regions.

\subsection{Mask R-CNN}

Mask R-CNN proposed by He in [19], is an instance object segmentation method that simultaneously generates detection and segmentation for each object in an input image, Step 1. This method consists of a parallel addendum to Faster R-CNN, a Fully Connected Network (FCN), which generates instance segmentation masks in addition to the classes, Step 4.a, and bounding boxes, Step 4.b, already detected by Faster R-CNN.

Faster R-CNN detection algorithm, on Step 2 of Figure 2, proposed in [38], is divided into two steps. The first is a Regional Proposal Network (RPN) that externals the candidate bounding boxes for each object. In the second stage, a Fast R-CNN generates the classification of each candidate object and improvement of its bounding box through regression.

The FCN, proposed in [26], on Step 3 of Figure 2, in subsequent to Faster R-CNN performs a Region of Interest (RoI) pixel-by-pixel classification, thus generating segmentation by instances in the image using a binary loss and sigmoid per pixel. The output of this network is the segmented object, Steps 5 and 6, region that best represents the spatial structure for this object in the image.

\subsection{Image Acquisition}

Seventeen echocardiogram exams were used, totaling 34 images, including end images diastole and systole. These images were taken of an echocardiograph (GE, Vivid 7 PRO model) of the Prontocardial Clinic in Fortaleza, CE. A echocardiographer (ECO 1) records seventeen exams synchronized by the electrocardiogram (ECG) left ventricular (LV) short axis position. The trackball is used to manually contours of the inner edges at the end of diastole and LV systole, synchronized with wave start Q and ECG T-wave peak, respectively, with immediate obtention of diastolic, systolic volume and ejection fraction (FEJ). Another 
echocardiographer (ECO 2) independently performs the measurements of the same 34 images on a laptop computer to interobserver analysis.

\subsection{Evaluation Metrics}

In this work, we use the following evaluation metrics for the segmentation performed: Accuracy (ACC) [12, 42], Sensitivity (Sen) [12, 42], Specificity (Spe) [12, 42], Positive Predictive Value (PPV) or Precision (PRE) [12, 42], F1-SCORE [42, 40], Matthews correlation coefficient (MCC) [28], Jaccard Index (JAC) [23] and Hausdorff Distante (HD) [20].

We calculated the metrics by comparing the segmentation pixels with the ground-truth pixels. Organ-owned pixels that were segmented as such are true positive values (TP), while pixels that do not belong but were segmented as part of the ventricle compute false positive values (FP) for non-segmented, non-organ pixels. Of interest, we have true negative values (TN), and when pixels that belong to the ventricle are not segmented, we get false negative values $(\mathrm{FN})$.

\section{An Automatic Left Ventricle Segmentation via Morphological Geodesic Active Contour with adaptive external energy}

In this section, we present a new approach to left ventricle segmentation inspired by classical geodesic active contour proposed by Caselles et al. [5], described in subsection 4.1, demonstrating its concepts and main equations. This approach is designed via morphological operators originally proposed by Marquez [27], described on subsection 4.2. Finally, in subsection 4.4, the proposed method is described based on previous fundamentals combined with an automatic initialization that approximates the initial contour of the desired segmentation region, providing rapid convergence, simplified and stable implementation.

\subsection{Traditional Geodesic Active Contour}

According to Caselles et al. [5] the evolution of a curve $C$ over time can be given due to the effect of the partial differential equations (PDE) $C_{t}=F \cdot N$, where $F$ denotes the curvature regarding normal vector. Previously, Osher-Sethian [33] proposed the representation of $C$ curve by means of a numerical scheme called Level Set. The Level Set method allows represent $C$ as a embedding function $u$, such that $C(t)=\{(x, y) ; u(t,(x, y))=0\}$ evolves in relation to time $t$. Thus, the curve fitting $C$ of any function $u(x, y)$ which embeds the curve as one of its level sets is:

$$
\frac{\partial u}{\partial t}=F \cdot|\nabla u|
$$

The mean curvature motion operator proposed by Evans et al. [11] give us curvature of $C$ :

$$
\frac{\partial u}{\partial t}=\operatorname{div}\left(\frac{\nabla u}{|\nabla u|}\right) \cdot|\nabla u|
$$

where the curvature of the implicit curve is given by the divergence of the normalized gradient. This representation aims to minimize the functional of energy from geodesic curve. The geodesic refer to differential geometry and means the shorter distance between two point on riemannian domain.

Based on this implicit representation of the curve $C$, Caselles et al. [5] proposed the traditional Geodesic Active Contour (GAC). The GAC method adds a border detector $g(I)$ that guides the curve evolution to the edge object:

$$
g(I)=\frac{1}{\sqrt{1+\alpha\left|\nabla G_{\sigma} * I\right|}}
$$


where $G_{\sigma} * I$ is a Gaussian filter with standard deviation $\sigma, \alpha$ is a weight factor. The $g(I)$ function is low near edges of image and allows us to select which regions of the image we are interested in.

In this sense, GAC method does not depend on the parametrization per point of the curve and the minimum energy curve corresponds to the geodesics of a riemannian space whose metric can be defined by $g(I)$. This energy minimization can be represent by:

$$
E(C)=\int_{0}^{\text {length }(C)} g(I)(C(s)) d s
$$

Euler-Lagrange equation can gives us the descent direction of the functional on 8:

$$
C_{t}=(g(I) K+g(I) v-\nabla g(I) \cdot N) N
$$

where $K$ is the euclidean curvature of $C, v \in \mathbb{R}$ is the balloon force parameter. Thus, is possible to define the curve evolution in a level set framework from the previous PDEs, rewriting Equation 9 in terms of level set:

$$
\begin{array}{r}
\frac{\partial u}{\partial t}=g(I)|\nabla u|\left(\operatorname{div}\left(\frac{\nabla u}{|\nabla u|}\right)+v\right)+\nabla g(I) \nabla u \\
+g(I) \cdot v \| \nabla u \mid
\end{array}
$$

Numerical integration methods can be used to solve differential equation at 10 , by means of finite-difference scheme. However, these techniques are computationally costly and converge slowly. In the next sections, we introduce an approach based on morphological operators in a simple, stable and fast way.

\subsection{A morphological approach to geodesic active contour}

Numerical solutions of partial differential equations were proposed by Lax [25]. His work was pioneer in the use successives mutiscale dilatations and erosions morphological [15], in order to offer a stable and efficient numerical scheme to solving PDEs. Posteriorly, Catté et al. [6] proved that successives applications of the mean operator, for a structuring element sufficiently small is equivalent to the curvature flow of the PDE at Equation 6.

Successive applications of dilatation and erosion morphological can achieve an equivalence relation with differential operators. Alvarez et al. [2] demonstrated that it is possible with a structuring element sufficiently small, i.e, $\lim _{m \rightarrow \infty}\left(D_{t / m}\right)^{m} m_{0}$. Thus, we can approximate the dilatation with infinitesimal equivalency to solution of $\frac{\partial u}{\partial t}=|\nabla u|$ as:

$$
\lim _{h \rightarrow 0^{+}} \frac{D_{h} u-u}{h}=|\nabla u|
$$

Similarly, the erosion operation can also approximated:

$$
\lim _{h \rightarrow 0^{+}} \frac{E_{h} u-u}{h}=-|\nabla u|
$$

with a result equivalent to solution of $\frac{\partial u}{\partial t}=-|\nabla u|$.

Marquez-Neila et al. [27] improved Lax curvature operator like a composition of morphological operators, $D E$ and $E D$. Therefore, by approximation, it is possible an equivalent behavior to the curvature operator (PDE 6) and contrast-invariant:

$$
\left(D E_{\sqrt{h}}\right) \circ\left(E D_{\sqrt{h}}\right) \approx \operatorname{div}\left(\frac{\nabla u}{|\nabla u|}\right) \cdot|\nabla u|
$$

where DE is ED are respectively the combination of erosion and morphological dilatation, and dilatation and morphological erosion, described by Marquez-Neila et al. [27]. 
Finally, inspired by equivalencies between morphological and differential operators, it is possible to solve the PDE 10 by means of approximations by morphological operators. Differential operators can be replaced by PDE 11 and PDE 12. The mean curvature motion PDE can be approximated by morphological curvature operator at Equation 13.

Moreover, in this morphological approach, it is noteworthy that the initialization level set refers to a binary component, where the curve representing that denotes the region of interest is defined by the edges of the of that component. A faster and stable way to converge the active contour, since level set binary is defined by a region very near to the target image edges. This approach requires a reduced number of iterations to achieve stable curve, offering a fast process of convergence.

In this sense, the proposed methodology is formed by the location stage of the left ventricle internal region for define the initial Level Set, subsection 4.4, and construction of the adaptive external energy that defines the evolution boundaries of the active contour, described in subsection 4.3).

\subsection{Adaptive External Energy}

This section presents the proposed approach to recognize the adaptive external energy. Two methods were evaluated to improve our methodology. The first, classical feature extractors are combined with classification models; second, deep learning approach using a classification technique based on regions. Figure 3 illustrates the step-by-step to recognize the ventricle region.

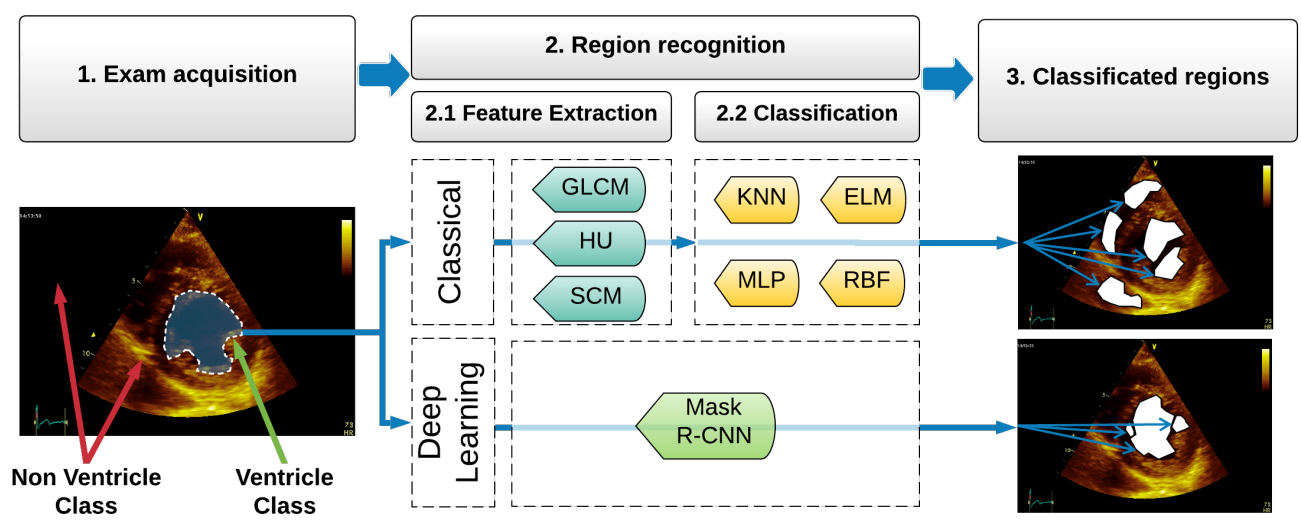

Figure 3. Flowchart to recognize the ventricle region. Above, the classical way that combines traditional feature extractors with classification models. Below, the approach based on deep learning.

\subsubsection{Classical approach}

The combination of classification models with traditional feature extractors formed the classical approach. This is useful to evaluate the capability of the traditional methods on the task of detecting cardiac muscle regions on echocardiogram exams.

\section{Feature extraction}

Three traditional feature extractors perform the stage of feature extraction based on image texture: GLCM, HU, and SCM. SCM method is evaluated under three configurations: Average, Gaussian, Laplacian, and Sobel Filter. Each feature extractor defined a vector that represents two different regions: Non-Ventricle Class and Ventricle Class. 


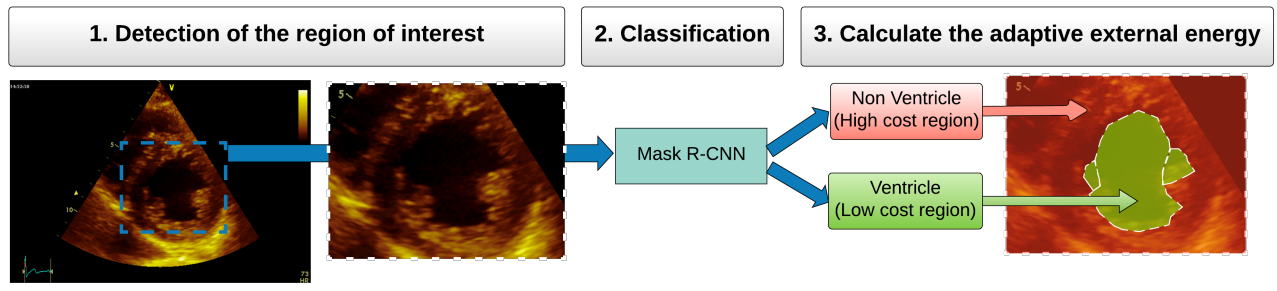

Figure 4. Adaptive external energy detection. To minimize the energy, only low-cost regions will be analyzed by FGAC [29].

\section{Classification}

After extract features, the output of the previous stage is used as input to the classifiers group: KNN, ELM, MLP, and RBF. The KNN model is configurated with $K$ varying from 1 to 5. ELM model is built with Moore-Penrose generalized inverse. MLP is configurated modifying its hidden layer with ten until hundred neurons. The dataset was split on $20 \%$ to test and $80 \%$ to train. All models were evaluated with 100 epochs, and average results were considered.

\subsubsection{Deep Learning Approach}

In this section, we will explain how we find the external energy that is used for the FGAC, describe the Mask R-CNN training step, and how we use its output as external energy for our method.

\section{Training Stage}

During training, we used Faster R-CNN Inception V2 as an attribute extractor for a batch of size 1 for 120,000 iterations. We consider an Inserction over Union (IoU) threshold of 0.9 to filter out low-confidence detections to ground-truth image. Initially, the learning rate was 0.002 being decreased by 10 into 90,000 steps. We use a momentum of 0.9 without Dropout.

The Total Loss for each RoI is given $L=L_{c l s}+L_{b o x}+L_{\text {mask }}$, where $L_{c l s}$ is the loss in classification, $L_{b o x}$ is the loss in the detection and $L_{\text {mask }}$ is the loss of the segmentation mask. $L_{\text {mask }}$ is used to generate masks for all classes, so the output mask is chosen from the class predicted by Faster R-CNN.

Training averaged 130 milliseconds per iteration. Total training time was 4 hours and 20 minutes, with a training set of $80 \%$ of the dataset with data augmentation images. We perform data augmentation by horizontally flipping the original images to double the training dataset from 28 to 56 images. At the end of the training, Total Loss was stabilized and less than $2 \%$.

\section{Energy Detection Stage}

From the segmented output instances of Mask R-CNN, we have made a binarization of the image, so we have separated the spatial structure that represents the ventricle within the image, as shown in Figure 2 at the output of the FCN. Then we extract the edge of the segmented image to use as External Energy for FGAC method.

Thus, from precisely segmented instances, we obtain profoundly relevant attributes of the ventricle edge, so that we have a more significant amount of information about this region of the organ, since it is not well defined in the image, avoiding possible failures in the segmentation targeting that are related to this region. 


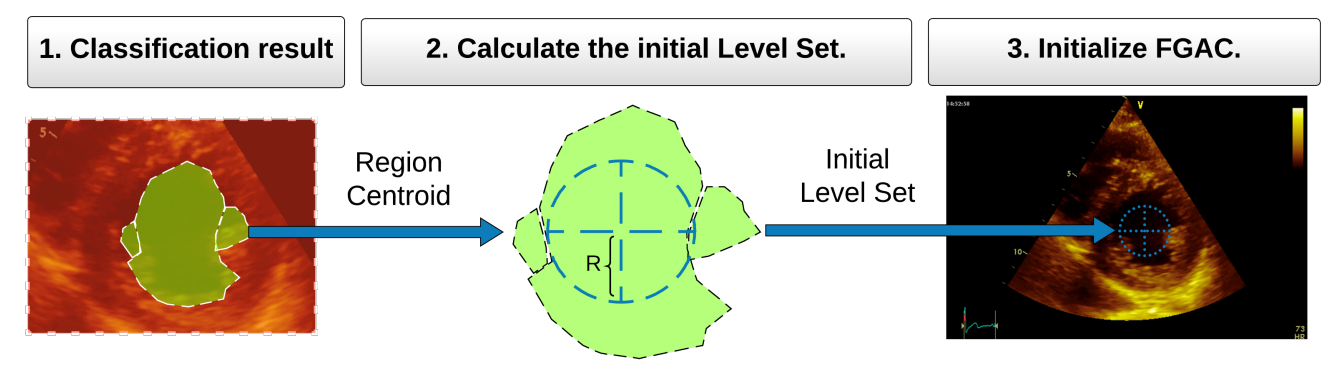

Figure 5. Flowchart for detection of the initial Level Set. An initial contour, with radius $R$, is projected with center at region centroid.

Figure 4 shows the flowchart of detection and calculation of adaptive external energy. After the result of Mask R-CNN, obtained in stage 2 of Figure 3, the area delimited by the region is attributed a negative cost, on the other hand, the external region represents an unwanted area and is assigned a positive cost. FGAC maintains its traditional evolution as proposed by Medeiros et al. [29], but we added the local cost calculation for the region. High-cost regions are considered as border regions. Thus the contour points do not advance in this region and continue to prioritize low-cost regions, searching for the gradient that defines the left ventricular border.

\subsection{Initial Level Set}

A crucial stage for approaches based on the active contour method is the definition of the initial contour. According to [37], initializations in undesired regions with low contrast contribute to the reduction of the influence of the gradient vector on the image, this may lead the method to early convergence or to define contours beyond the region of interest.

This work proposes an adaptive initialization based on the local classification of regions of interest. The methodology considered two different approaches. The first initialization, according to region attribute extraction that defines the left ventricular area, this step is followed by classification using traditional classification models. The second approach, however, uses the convolutional neural network Mask R-CNN mentioned on section 4.4.

Figure 5 illustrates the stages of initial contour detection. At step 1, the echocardiogram exam is linked, then at stage 2, two approaches for detecting candidate regions are evaluated. In stage 3, the classifiers identify the candidate regions. Finally, in step 4, the centroid of the candidate region is calculated. From this centroid with radius $R$, the initial level set of the active geodetic contour is identified.

\section{Results}

This section describes the results of the proposed approach, which were calculated on a machine that comprises an Intel i7, 8GB of memory, with the Linux Ubuntu 16.04 operating system. The results in this paper are shown in three stages. The first step corresponds to the study on the calculation of adaptive external energy, evaluating two approaches, one traditional and the other with deep learning. The second stage evaluates FGAC performance combined with adaptive energy for left ventricular segmentation. Finally, a comparison to the expert methods Radial snakes with derivative (RSD), Radial snakes with Hilbert energy (RSH) [1], and pSnakes based polar coordinates [9] is showed.

According the results using classical approaches, presented in Table 1, the traditional approach for the calculation of adaptive external energy presented hit rates close to $60 \%$, in contrast with the combination of the SCM characteristic extractor with Sobel filter combined with the RBF classifier that presented an accuracy above $83.56 \%$. Besides, the 
best combination also had a sensitivity higher than $87.73 \%$, which indicates a considerable approximation of the desired region.

Looking at Table 3, we have the combinations of extraction methods with the Bayesian classifier as the fastest, with classification time about 0.055 seconds to the SCM-Average extractor. However, as seen in Table 1, its hit rate presented a low performance, with $61.91 \%$ for the fastest combination. Meanwhile, the combination of best metrics, according to Table 1, was obtained by RBF and SCM-Sobel, achieving a high ranking time, about 7.247 seconds. The Figure 6 presents a comparison between the best combinations of classical extractors and classifier models.

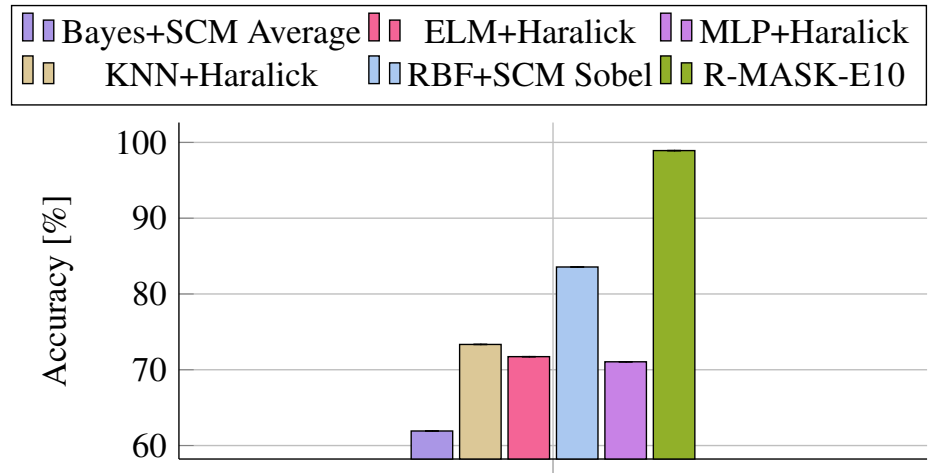

Best classifier with feature extractor.

Figure 6. Graph representation of the best classifier combinations and feature extractor against the deep learning model R-MASK.

On other hand, according to Table 2, we have Mask R-CNN-E10 with metrics higher than the RBF and SCM-Sobel combination, presenting $98.91 \%$ of accuracy and $99.93 \%$ of specificity, with classification time equals to 1.946 seconds, almost four times faster. So we can say that our adaptive external energy approach using Mask R-CNN-E10 is more efficient than using classic machine learning and extraction methods. The hit rates have average values above $98.91 \%$, and for Specificity, the method has a rate of $99.93 \%$ indicating a high hit rate of the method relative to the regions of interest that represent the left ventricle. The results suggest that the calculation of adaptive external energy by R-MASK presents a better sensitivity to recognize the left ventricular area, surpassing all other classical combinations according to Figure 6.

Finally, by evaluating FGAC performance with echocardiographic imaging expert methods, according to the manual segmentation by two human specialists, echocardiographer 1 (ECO1) and echocardiographer 2 (ECO2). The results indicate that the proposed approach presents a higher accuracy over the ECO1 expert markings, averaging $99.53 \% \pm 0.47$ outperforming the pSnakes, RSD, and RSH methods with $99.52 \% \pm 0.38$, $99.46 \% \pm 0.40$, and $99.51 \% \pm 0.37$, respectively. Similarly, the same result was also presented when comparing the manual ECO2 segmentations, according to Table 4. A visual comparison is presented in Figure 7, showing that the proposed approach achieved a precision higher than other segmentation methods, according to manual segmentation made for both specialists.

Two segmentation samples via FGAC combined with the adaptive external energy can be seen in Figure 8, Figures 8(a) and 8(b) show contour comparisons between specialist and proposed segmentation. The green contour represents the specialist segmentation while the white contour represents the proposed segmentation. In addition to the evaluation metrics already mentioned, the visual approximation between both contours corroborates to previous results. 


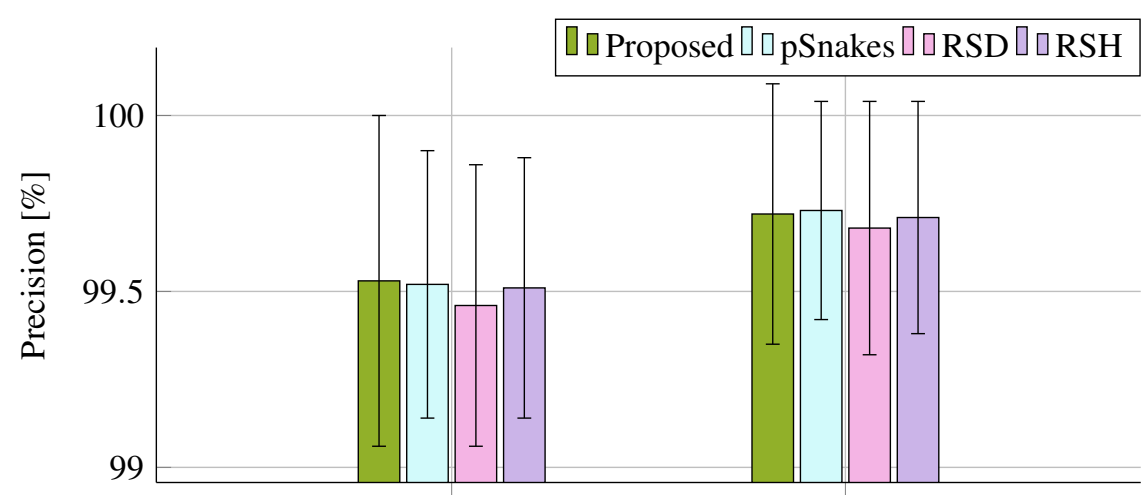

Echocardiographer $1 \quad$ Echocardiographer 2

Approachs

Figure 7. Graph representation of the Precision metric achieved by the approach proposed according to manual segmentation from two echocardiographers.

Table 1. Accuracy, Sensitivity, Specificity, PPV, F1-Score, and MCC obtained through the classification of extracted attributes with classical extractors.

\begin{tabular}{|c|c|c|c|c|c|c|c|}
\hline \multicolumn{2}{|r|}{ Method } & $\mathbf{A C C}$ & Sen & Spe & PPV & F1-SCORE & MCC \\
\hline \multirow{6}{*}{ Bayes } & Haralick & 28.97 & 71.45 & 27.97 & 2.28 & 4.42 & 0.19 \\
\hline & $\mathrm{Hu}$ & 43.21 & 71.44 & 42.58 & 2.68 & 5.17 & 4.13 \\
\hline & SCM-Average & 61.92 & 60.38 & 61.95 & 3.37 & 6.39 & 6.66 \\
\hline & SCM-Gaussian & 58.87 & 64.56 & 58.75 & 3.33 & 6.33 & 6.86 \\
\hline & SCM-Laplacian & 48.57 & 86.09 & 47.73 & 3.55 & 6.81 & 9.90 \\
\hline & SCM-Sobel & 51.77 & 80.78 & 51.13 & 3.53 & 6.76 & 9.29 \\
\hline \multirow{6}{*}{$K \mathrm{NN}$} & Haralick & 73.34 & 67.25 & 73.48 & 5.34 & 9.90 & 13.33 \\
\hline & $\mathrm{Hu}$ & 61.01 & 79.86 & 60.59 & 4.26 & 8.10 & 11.97 \\
\hline & SCM-Average & 59.69 & 80.26 & 59.24 & 4.16 & 7.91 & 11.64 \\
\hline & SCM-Gaussian & 60.47 & 78.96 & 60.06 & 4.17 & 7.92 & 11.52 \\
\hline & SCM-Laplacian & 64.90 & 79.82 & 64.57 & 4.80 & 9.06 & 13.50 \\
\hline & SCM-Sobel & 65.27 & 79.52 & 64.95 & 4.76 & 8.99 & 13.46 \\
\hline \multirow{6}{*}{ ELM } & Haralick & 71.73 & 68.04 & 71.81 & 5.09 & 9.47 & 12.80 \\
\hline & $\mathrm{Hu}$ & 43.21 & 78.42 & 42.41 & 3.00 & 5.78 & 6.22 \\
\hline & SCM-Average & 67.39 & 62.04 & 67.51 & 4.03 & 7.57 & 9.11 \\
\hline & SCM-Gaussian & 66.81 & 63.12 & 66.89 & 4.03 & 7.57 & 9.21 \\
\hline & SCM-Laplacian & 61.96 & 83.17 & 61.49 & 4.57 & 8.66 & 13.30 \\
\hline & SCM-Sobel & 61.77 & 81.33 & 61.33 & 4.43 & 8.41 & 12.68 \\
\hline \multirow{6}{*}{$\mathbf{R B F}$} & Haralick & 58.61 & 59.62 & 58.59 & 3.41 & 6.46 & 5.65 \\
\hline & $\mathrm{Hu}$ & 51.51 & 87.51 & 50.56 & 4.47 & 8.50 & 12.06 \\
\hline & SCM-Average & 72.72 & 53.65 & 73.12 & 4.07 & 7.56 & 8.57 \\
\hline & SCM-Gaussian & 73.95 & 48.90 & 74.55 & 4.45 & 8.16 & 8.13 \\
\hline & SCM-Laplacian & 68.20 & 85.25 & 67.77 & 6.21 & 11.57 & 17.33 \\
\hline & SCM-Sobel & 83.56 & 87.74 & 83.41 & 16.44 & 27.69 & 33.63 \\
\hline \multirow{6}{*}{ MLP } & Haralick & 71.05 & 70.33 & 71.07 & 5.15 & 9.59 & 13.22 \\
\hline & $\mathrm{Hu}$ & 52.14 & 79.69 & 51.51 & 3.62 & 6.93 & 9.23 \\
\hline & SCM-Average & 64.77 & 64.45 & 64.78 & 3.88 & 7.32 & 8.86 \\
\hline & SCM-Gaussian & 65.22 & 65.11 & 65.22 & 3.96 & 7.46 & 9.20 \\
\hline & SCM-Laplacian & 62.66 & 82.84 & 62.20 & 4.67 & 8.89 & 13.56 \\
\hline & SCM-Sobel & 63.48 & 81.57 & 63.08 & 4.68 & 8.84 & 13.42 \\
\hline
\end{tabular}


Table 2. Accuracy, Sensitivity, Specificity, PPV, F1-Score, and MCC obtained through the classification of R-MASK CNN combined with region adjusting.

\begin{tabular}{c|c|c|c|c|c|c}
\hline Method & ACC & Sen & Spe & PPV & F1-SCORE & MCC \\
\hline \hline R-MASK-E5 & 98.85 & 70.93 & 99.80 & 92.47 & 80.28 & 80.45 \\
\hline R-MASK-E10 & $\mathbf{9 8 . 9 2}$ & $\mathbf{6 8 . 5 3}$ & $\mathbf{9 9 . 9 4}$ & $\mathbf{9 7 . 2 4}$ & $\mathbf{8 0 . 4 0}$ & $\mathbf{8 1 . 1 6}$ \\
\hline R-MASK-E11 & 98.33 & 49.25 & 99.97 & 98.41 & 65.64 & 69.00 \\
\hline \hline
\end{tabular}

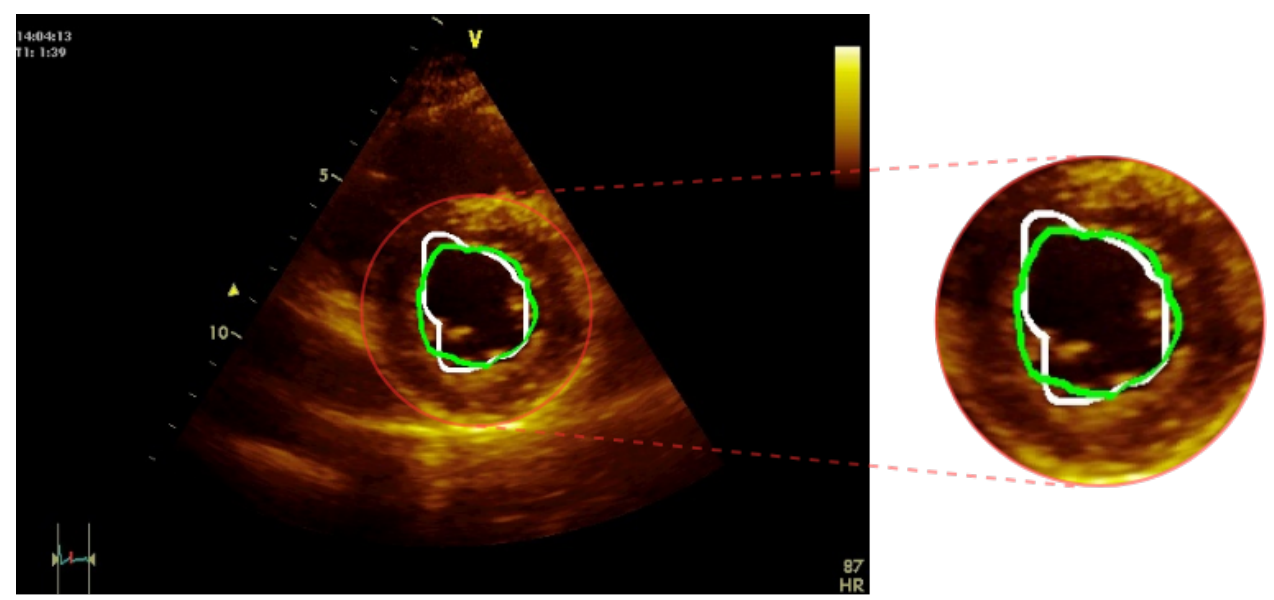

(a) Echocardiogram 1.
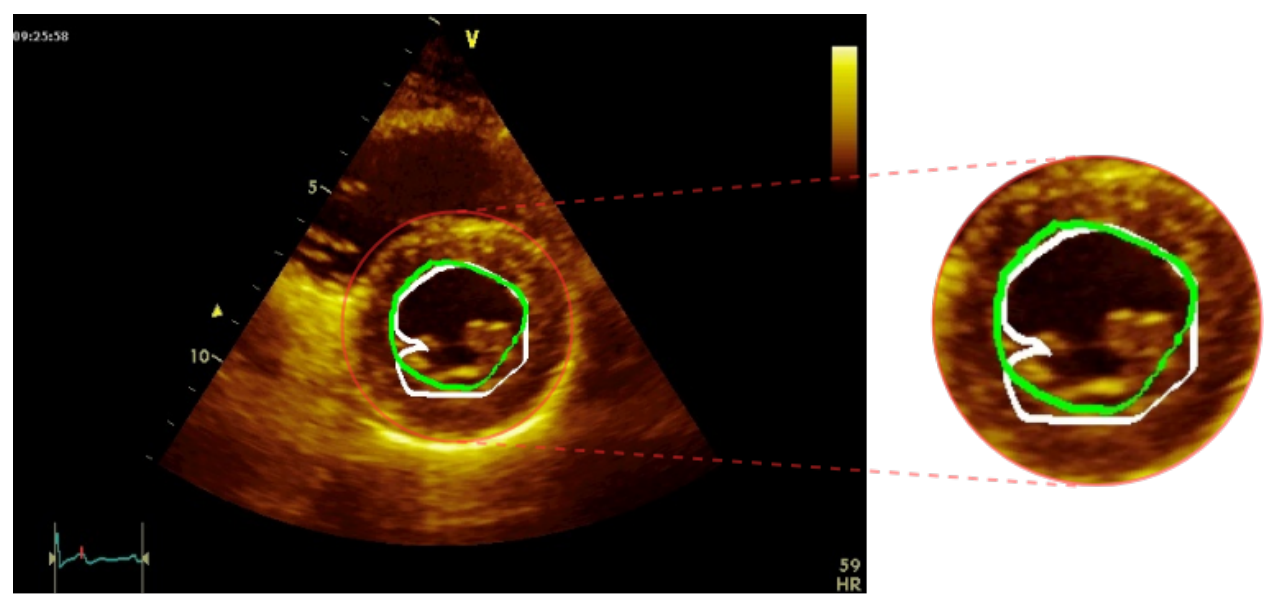

(b) Echocardiogram 2.

Figure 8. Contour images of ground truth (green) and FGAC output (white) of the left ventricle segmentation. 
Table 3. Average classification time to evaluated methods.

\begin{tabular}{|c|c|c|}
\hline \multicolumn{2}{|c|}{ Method } & Classification Time (s) \\
\hline \multirow{6}{*}{ Bayes } & Haralick & 0.0749179 \\
\hline & $\mathrm{Hu}$ & 0.0581979 \\
\hline & SCM-Average & 0.0553418 \\
\hline & SCM-Gaussian & 0.0577158 \\
\hline & SCM-Laplacian & 0.0567364 \\
\hline & SCM-Sobel & 0.0561102 \\
\hline \multirow{6}{*}{ KNN-5 } & Haralick & 11.2853684 \\
\hline & $\mathrm{Hu}$ & 9.0762435 \\
\hline & SCM-Average & 9.1499707 \\
\hline & SCM-Gaussian & 9.0275925 \\
\hline & SCM-Laplacian & 9.1266493 \\
\hline & SCM-Sobel & 9.1100188 \\
\hline \multirow{6}{*}{ ELM } & Haralick & 1.0292084 \\
\hline & $\mathrm{Hu}$ & 1.1189812 \\
\hline & SCM-Average & 1.0331317 \\
\hline & SCM-Gaussian & 1.0582434 \\
\hline & SCM-Laplacian & 1.0497182 \\
\hline & SCM-Sobel & 1.0708105 \\
\hline \multirow{6}{*}{$\mathrm{RBF}$} & Haralick & 10.0292491 \\
\hline & $\mathrm{Hu}$ & 7.2972785 \\
\hline & SCM-Average & 7.2283257 \\
\hline & SCM-Gaussian & 7.2405187 \\
\hline & SCM-Laplacian & 7.2616954 \\
\hline & SCM-Sobel & 7.2470709 \\
\hline \multirow{6}{*}{ MLP } & Haralick & 2.3964001 \\
\hline & $\mathrm{Hu}$ & 2.4210172 \\
\hline & SCM-Average & 2.3627517 \\
\hline & SCM-Gaussian & 2.3352131 \\
\hline & SCM-Laplacian & 2.2789647 \\
\hline & SCM-Sobel & 2.2695069 \\
\hline \multicolumn{2}{|c|}{ CNN-RMASK } & 1.9460632 \\
\hline
\end{tabular}

Table 4. Proposed approach result against available segmentation methods in the specialized literature.

\begin{tabular}{c|c|c|c|c|c|c}
\hline Expert & Method & PRE & ACC & JAC & HD & SPC \\
\hline \hline ECO1 & Proposed & $\mathbf{9 9 . 5 3} \pm \mathbf{0 . 4 7}$ & $\mathbf{9 8 . 6 4} \pm \mathbf{0 . 5 1}$ & $\mathbf{6 7 . 4} \pm \mathbf{1 1 . 8 2}$ & $\mathbf{5 . 6 4} \pm \mathbf{0 . 9 7}$ & $\mathbf{9 9 . 0 7} \pm \mathbf{0 . 5 1}$ \\
\hline ECO1 & pSnakes $[9]$ & $99.52 \pm 0.38$ & $99.02 \pm 0.55$ & $74.34 \pm 12.07$ & $4.90 \pm 1.14$ & $99.46 \pm 0.54$ \\
\hline ECO1 & RSD $[1]$ & $99.46 \pm 0.40$ & $99.07 \pm 0.49$ & $74.32 \pm 11.76$ & $4.65 \pm 1.05$ & $99.57 \pm 0.51$ \\
\hline ECO1 & RSH $[1]$ & $99.51 \pm 0.37$ & $99.05 \pm 0.59$ & $74.46 \pm 12.18$ & $4.80 \pm 1.13$ & $99.51 \pm 0.64$ \\
\hline \hline ECO2 & Proposed & $\mathbf{9 9 . 7 2} \pm \mathbf{0 . 3 7}$ & $\mathbf{9 8 . 4 6} \pm \mathbf{0 . 6 5}$ & $\mathbf{6 2 . 0 2} \pm \mathbf{1 5 . 2 4}$ & $\mathbf{5 . 6 6} \pm \mathbf{1 . 1 6}$ & $\mathbf{9 8 . 6 9} \pm \mathbf{0 . 7 3}$ \\
\hline ECO2 & pSnakes $[9]$ & $99.73 \pm 0.31$ & $98.86 \pm 0.66$ & $69.22 \pm 14.20$ & $5.17 \pm 1.14$ & $99.10 \pm 0.71$ \\
\hline ECO2 & RSD $[1]$ & $99.68 \pm 0.36$ & $98.94 \pm 0.62$ & $70.00 \pm 13.01$ & $5.01 \pm 1.08$ & $99.22 \pm 0.68$ \\
\hline ECO2 & RSH $[1]$ & $99.71 \pm 0.33$ & $98.88 \pm 0.75$ & $69.36 \pm 13.31$ & $5.20 \pm 1.25$ & $99.14 \pm 0.81$ \\
\hline \hline
\end{tabular}




\section{Conclusion}

In this paper, we propose a new approach to automatic segmentation of the left ventricle in the echocardiogram exams. In this approach, we combine the morphological geodesic active contour with a new adaptive external energy based on classification via deep learning technique. To automatically identify the left ventricle region, the results indicated that the classical approach achieves its best result with SCM-Sobel combined with the RBF model, performing a hit rate of about $83.56 \%$. On the other hand, the deep learning technique via Mask R-CNN presented the best hit rate, with $98.92 \%$. This method surpasses all other combinations, indicating its applicability in the calculus of the adaptive external energy.

The addition of adaptive external energy presented the hit rate equivalent to or higher than the expert methods already shown in the literature, with promising higher accuracy $(99.72 \%)$ and equivalents precision $(99.72 \%)$ and similarity rates as Jaccard Index $(67.40 \%)$ and HD (5.6384). These results suggest that the adaptive approach can to aid the FGAC in differentiating the cardiac muscular tissue of other regions, achieving promising performance.

As future works, we aim to apply the method to a dataset with higher image volume, aiming to improve the prediction of adaptive external energy with Mask R-CNN and other new deep learning techniques based on region classification. Besides, we aim to evaluate the performance of the method to calculate the ventricular ejection fraction, mainly when the region is damaged by pathologies that altered the image contrast.

\section{Acknowledgments}

This study was financed in part by the Coordenação de Aperfeiçoamento de Pessoal de Nível Superior - Brasil (CAPES) - Finance Code 001”. Also Pedro Pedrosa Rebouças Filho acknowledges the sponsorship from the Brazilian National Council for Research and Development (CNPq) via Grants Nos. 431709/2018-1 and 311973/2018-3.

\section{Conflicts of Interest}

There is no conflict of interest.

\section{References}

[1] AR Alexandria, Paulo César Cortez, JHS Felix, TS Cavalcante, PP Rebouças Filho, JAC Silva Júnior, and JS Abreu. Hilbertian energy: a method for external energy calculation on radial active contours. In 17th International conference on systems, signals and image processing-IWSSIP 2010, 2010.

[2] Luis Alvarez, Frédéric Guichard, Pierre-Louis Lions, and Jean-Michel Morel. Axioms and fundamental equations of image processing. Archive for rational mechanics and analysis, 123(33):199-257, 1993.

[3] S Avinash, K Manjunath, and S Senthil Kumar. An improved image processing analysis for the detection of lung cancer using gabor filters and watershed segmentation technique. In International Conference on Inventive Computation Technologies (ICICT), volume 3, pages 1-6. IEEE, 2016.

[4] Jéssyca Almeida Bessa, Paulo César Cortez, John Hebert da Silva Félix, Ajalmar Rêgo da Rocha Neto, and Auzuir Ripardo de Alexandria. Radial snakes: comparison of segmentation methods in synthetic noisy images. Expert Systems with Applications, 42(6):3079-3088, 2015. 
[5] Vicent Caselles, Ron Kimmel, and Guillermo Sapiro. Geodesic active contours. International journal of computer vision, 22(1):61-79, 1997.

[6] Francine Catté, Françoise Dibos, and Georges Koepfler. A morphological scheme for mean curvature motion and applications to anisotropic diffusion and motion of level sets. SIAM Journal on Numerical Analysis, 32(6):1895-1909, 1995.

[7] Timothy F. Cootes, Gareth J. Edwards, and Christopher J. Taylor. Active appearance models. IEEE Transactions on pattern analysis and machine intelligence, 23(6): 681-685, 2001.

[8] John Hebert da Silva Felix, Paulo C. Cortez, Rodrigo C. S. Costa, Simone C. B. Fortaleza, et al. Avaliação computacional de enfisema pulmonar em TC: comparação entre um sistema desenvolvido localmente e um sistema de uso livre. Jornal Brasileiro de Pneumologia, 35(9):868-876, 2009.

[9] Auzuir Ripardo De Alexandria, Paulo César Cortez, Jessyca Almeida Bessa, John Hebert da Silva Félix, José Sebastião De Abreu, and Victor Hugo C De Albuquerque. psnakes: A new radial active contour model and its application in the segmentation of the left ventricle from echocardiographic images. Computer methods and programs in biomedicine, 116(3):260-273, 2014.

[10] Antonio de Padua Mansur and Desidério Favarato. Mortalidade por doenças cardiovasculares em mulheres e homens nas cinco regiões do brasil, 1980-2012. Arquivos Brasileiros de Cardiologia, 107(2):137-146, 2016.

[11] Lawrence C Evans, Joel Spruck, et al. Motion of level sets by mean curvature. i. Journal of Differential Geometry, 33(3):635-681, 1991.

[12] Tom Fawcett. An introduction to roc analysis. Pattern recognition letters, 27(8): 861-874, 2006.

[13] K. Fukunaga and P. M. Narendra. A branch and bound algorithm for computing k-nearest neighbors. IEEE Transactions on Computers, C-24(7):750-753, July 1975. ISSN 0018-9340. .

[14] Selena Gonzales and Bradley Sawyer. How do mortality rates in the u.s. compare to other countries? Kaiser Family Found, 2017. URL http://ww.healthsystemtracker.org/chart-collection/ mortality-rates-u-s-compare-countries/?_sf_s=mortality\# item-start. Online on: 08/07/2017.

[15] Rafael C Gonzalez. Digital image processing. Pearson Education India, 2009.

[16] Yanhui Guo, Guo-Qing Du, Jing-Yi Xue, Rong Xia, and Yu-hang Wang. A novel myocardium segmentation approach based on neutrosophic active contour model. Computer methods and programs in biomedicine, 142:109-116, 2017.

[17] Robert M Haralick, Karthikeyan Shanmugam, et al. Textural features for image classification. IEEE Transactions on systems, man, and cybernetics, 3(6):610-621, 1973.

[18] Simon Haykin. Neural Networks and Learning Machines. Prentice Hall, McMaster University, Canada, 2008.

[19] Kaiming He, Georgia Gkioxari, Piotr Dollár, and Ross Girshick. Mask r-cnn. In Proceedings of the IEEE international conference on computer vision, pages 29612969, 2017. 
[20] Jeff Henrikson. Completeness and total boundedness of the hausdorff metric. MIT Undergraduate Journal of Mathematics, 1:69-80, 1999.

[21] Ming-Kuei Hu. Visual pattern recognition by moment invariants. IRE transactions on information theory, 8(2):179-187, 1962.

[22] Guang-Bin Huang, Qin-Yu Zhu, Chee-Kheong Siew, et al. Extreme learning machine: a new learning scheme of feedforward neural networks. Neural networks, 2:985-990, 2004.

[23] Paul Jaccard. Étude comparative de la distribution florale dans une portion des alpes et des jura. Bull Soc Vaudoise Sci Nat, 37:547-579, 1901.

[24] Michael Kass, Andrew Witkin, and Demetri Terzopoulos. Snakes: Active contour models. In International Journal of Computer Vision, volume 1, pages 321-331, 1987.

[25] PD Lax. Numerical solution of partial differential equations. The American Mathematical Monthly, 72(sup2):74-84, 1965.

[26] Jonathan Long, Evan Shelhamer, and Trevor Darrell. Fully convolutional networks for semantic segmentation. In Proceedings of the IEEE conference on computer vision and pattern recognition, pages 3431-3440, 2015.

[27] Pablo Marquez-Neila, Luis Baumela, and Luis Alvarez. A morphological approach to curvature-based evolution of curves and surfaces. IEEE Transactions on Pattern Analysis and Machine Intelligence, 36(1):2-17, 2014.

[28] Brian W Matthews. Comparison of the predicted and observed secondary structure of t4 phage lysozyme. Biochimica et Biophysica Acta (BBA)-Protein Structure, 405(2): 442-451, 1975.

[29] Aldísio G Medeiros, Matheus T Guimarães, Solon A Peixoto, Lucas de O Santos, Antônio C da Silva Barros, Elizângela de S Rebouças, Victor Hugo C de Albuquerque, and Pedro P Rebouças Filho. A new fast morphological geodesic active contour method for lung ct image segmentation. Measurement, 2019.

[30] Aldisio Gonçalves Medeiros and Daniel S. Ferreira. Detecção de agrupamentos de microcalcificações em imagens digitais de mamografias. XV WIM - Workshop de Informática Médica, Recife - PE, 2015.

[31] S Nandagopalan, BS Adiga, C Dhanalakshmi, and N Deepak. Automatic segmentation and ventricular border detection of $2 \mathrm{~d}$ echocardiographic images combining k-means clustering and active contour model. In Computer and Network Technology (ICCNT), 2010 Second International Conference on, pages 447-451. IEEE, 2010.

[32] Melanie Nichols, Nick Townsend, Peter Scarborough, and Mike Rayner. Cardiovascular disease in europe 2014: epidemiological update. European Heart Journal, 35(42):2950, 2014. . URL +http://dx. doi .org/10.1093/eurheartj/ ehu299. Acessado em: 08/07/2017.

[33] Stanley Osher and James A Sethian. Fronts propagating with curvature-dependent speed: algorithms based on hamilton-jacobi formulations. Journal of computational physics, 79(1):12-49, 1988.

[34] Jooyoung Park and Irwin W Sandberg. Universal approximation using radial-basisfunction networks. Neural computation, 3(2):246-257, 1991.

[35] Caroline Petitjean and Jean-Nicolas Dacher. A review of segmentation methods in short axis cardiac mr images. Medical image analysis, 15(2):169-184, 2011. 
[36] Geraldo L Bezerra Ramalho, Daniel S Ferreira, Pedro P Rebouças Filho, and Fátima N Sombra de Medeiros. Rotation-invariant feature extraction using a structural cooccurrence matrix. Measurement, 94:406-415, 2016.

[37] Pedro Pedrosa Rebouças Filho, Paulo C. Cortez, Antônio C. S. Barros, and Victor H. C. De Albuquerque. Novel adaptive balloon active contour method based on internal force for image segmentation-A systematic evaluation on synthetic and real images. Expert Systems with Applications, 41(17):7707-7721, 2014.

[38] Shaoqing Ren, Kaiming He, Ross Girshick, and Jian Sun. Faster r-cnn: Towards real-time object detection with region proposal networks. In Advances in neural information processing systems, pages 91-99, 2015.

[39] Gustavo Rolando, Emilio Daniel Valenzuela Espinoza, Emelin Avid, Sebastián Welsh, et al. Prognostic value of ventricular diastolic dysfunction in patients with severe sepsis and septic shock. Revista Brasileira de terapia intensiva, 27(4):333-339, 2015.

[40] Yutaka Sasaki et al. The truth of the f-measure. Teach Tutor mater, 1(5):1-5, 2007.

[41] D Serre. Matrices: Theory and applications. 2002. Graduate texts in mathematics, 2000.

[42] Marina Sokolova and Guy Lapalme. A systematic analysis of performance measures for classification tasks. Information processing \& management, 45(4):427-437, 2009.

[43] Sergios Theodoridis and Konstantinos Koutroumbas. Pattern Recognition (Fourth Edition). Academic Press, USA, 2008. 